\title{
Directional telomeric silencing and lack of canonical $B 1$ elements in two silencer Autonomously Replicating Sequences in S. cerevisiae
}

\author{
Patricia Chisamore-Robert ${ }^{1}$, Samantha Peeters ${ }^{2}$, Kristina Shostak ${ }^{1}$ and Krassimir Yankulov ${ }^{1 *}$
}

\begin{abstract}
Background: Autonomously Replicating Sequences (ARS) in S. cerevisiae serve as origins of DNA replication or as components of cis-acting silencers, which impose positional repression at the mating type loci and at the telomeres. Both types of ARS can act as replicators or silencers, however it is not clear how these quite diverse functions are executed. It is believed that all ARS contain a core module of an essential ARS Consensus Sequence (ACS) and a non-essential B1 element.

Results: We have tested how the B1 elements contribute to the silencer and replicator function of ARS. We report that the ACS-B1 orientation of ARS has a profound effect on the levels of gene silencing at telomeres. We also report that the destruction of the canonical B1 elements in two silencer ARS (ARS317 and ARS319) has no effect on their silencer and replicator activity.

Conclusions: The observed orientation effects on gene silencing suggest that ARSs can act as both proto-silencers and as insulator elements. In addition, the lack of B1 suggests that the ACS-B1 module could be different in silencer and replicator ARS.
\end{abstract}

Keywords: Autonomously replicating sequences, Telomere position effect, DNA replication, Gene silencing

\section{Background}

Origins of DNA replication in budding yeast are well defined DNA elements referred to as Autonomously Replicating Sequences (ARS). They consist of a core 11 base pair ACS (ARS Consensus Sequence, WTTTAYRTTTW) and three or four auxiliary $\underline{B}$ elements [1]. ACS is critical for the function of ARS [2-4]. It is the main site of binding of the Origin Recognition Complex (ORC), which nucleates the formation of pre-replicative complexes in G1 $[5,6]$. It has been shown that the flanking sequences of the core $A C S$ can also contribute to the binding of ORC thus producing the 17 base pair extended ACS (EACS) $[7,8]$. Compared to $A C S$, the $\underline{B}$ elements are not so well characterized and their roles are poorly understood. $\underline{B 2}$ is believed to act as a site of DNA unwinding that allows for the initiation of replication $[9,10] . \underline{B 3}$ is a binding site for Abf1p [11]. While the significance of these elements

\footnotetext{
* Correspondence: yankulov@uoguelph.ca

'Department of Molecular and Cellular Biology, University of Guelph, Guelph, ON, Canada

Full list of author information is available at the end of the article
}

for the activity of the origins is apparent, the mechanism of their action is unclear. The $\underline{B 1}$ element is positioned about 15 bases upstream of the core ACS. Using ARS1 as a model, it has been shown that $\underline{B 1}$ acts as a second binding site for ORC $[5,6]$. Earlier studies have proposed an AWnY consensus 14 bases upstream of ACS [12]. A more comprehensive analysis and alignment of multiple origins has shown better agreement for a WTW motif positioned 15-17 bases upstream of the core ACS [8]. Mutations in these WTW motifs have substantially reduced the replicator activity of most of the tested origins $[8,13]$. It has been proposed that the $B 1$ element together with the extended $A C S$ produce a variety of bi-partite sites that bind ORC with different affinities $[7,12,14]$.

Besides their role in DNA replication, ARS and ORC play a central role in gene silencing at the constitutively repressed mating type loci $H M L$ and $H M R$ and in the subtelomeric regions of the chromosomes [1]. The silencers, which flank $H M L$ and $H M R$ and impose complete shut-off of the genes between them, are built up of various combinations of binding sites for Abf1p and Rap1p 
plus one of four ARSs (ARS301, ARS302, ARS317, ARS318) [1]. Mutations in the ACS of these ARSs substantially reduce gene repression and confer inability to mate [1]. Interestingly, at the $H M L-I$ and $H M R-E$ silencers the orientation of the $A C S-B 1$ elements of the $A R S s$ has a directional effect on the levels of gene repression [15-17]. Multiple ARSs are also found in the core $X$ and $Y^{\prime}$ subtelomeric regions $[18,19]$. At these positions they act as proto-silencers meaning that they relay and enhance the repression signals emitted by the telomeres [20]. It is not known if these ARSs have directional function. The ARSs at the subtelomeric and the mating type loci rarely fire at their native locations thus strengthening the notion that they have a silencing function independent of the initiation of DNA replication. However, when moved to a mini-chromosome or at different genomic position, these ARSs can fire as efficiently as any other $A R S[21,22]$. The basis of this dual function of $A R S s$ is not fully understood [21].

Recent studies have shown that targeted mutations in the putative $\underline{B 1}$ (WTW) motifs of certain ARSs have little effect on their replicator activity $[4,8]$. It is unclear if the same mutations affect gene silencing. It remains possible that such $A R S s$ contain a $\underline{B 1}$ at a different position. Ultimately, it is unclear if $\underline{B 1}$ plays a role in $A R S$ dependent gene silencing. In this study we have tested the role of $\underline{B 1}$ by parallel silencing and replicator assays. We have found that the putative $\underline{B 1}$ elements of two silencer ARSs (ARS317 and ARS319) are dispensable for both activities.

\section{Results}

\section{Experimental strategy}

To address the role of $\underline{B 1}$ in gene silencing, we isolated two silencer $A R S$ (ARS317 from the mating type $H M R$ locus and $A R S 319$ from the IIIR subtelomeric region) and two well-characterized replicator ARS (ARS305 and $A R S 605)$. These were inserted next to the VIIL telomere in $A C S-B 1$ and $B 1-A C S$ orientation (Figure 1) and the silencing of the adjacent $U R A 3$ was assessed. In addition, we performed scanning mutagenesis of the $\underline{B 1}$ elements of these $A R S s$ and estimated the effects of the mutations on their silencer and replicator activity (Figure 2, Figure 3).

The silencer activity of all the ARSs was assessed by a routine TPE (Telomere Position Effect) assay [23]. The rationale of the assay is as follows: Telomeres recruit multiple Rap1 proteins, which in turn recruit Sir2/3/4 proteins $[24,25]$. The Sir proteins then spread over and de-acetylate the neighboring nucleosomes to establish a heterochromatin domain [1]. Depending on the scope of spreading, subtelomeric genes are either active or completely repressed and infrequently switch between the two states [23]. When $U R A 3$ is inserted at the VIIL

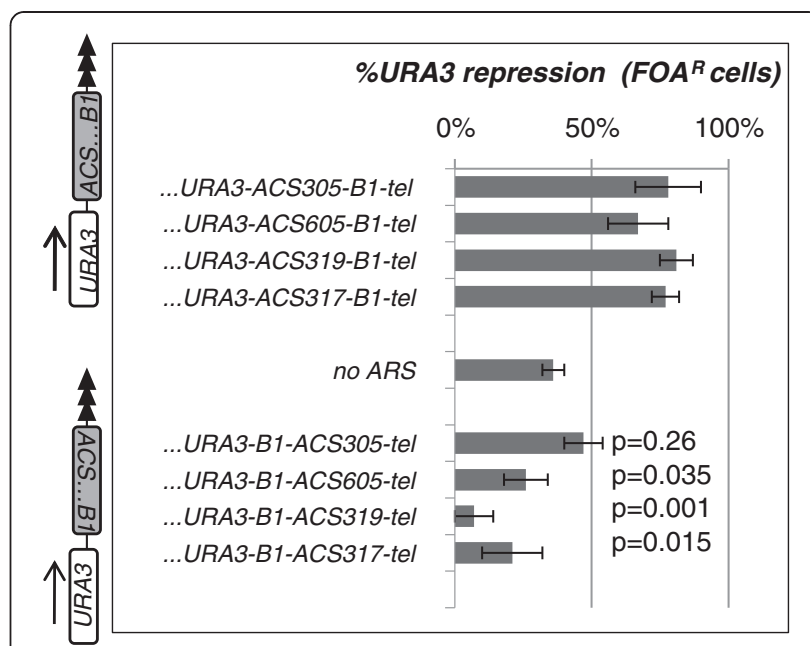

Figure 1 Orientation-dependent silencing by ARSs. ARS305, ARS605, ARS317 and ARS319 were cloned in URA3-ACS-B1-tel (upper part) and URA3-B1-ACS-tel (lower part) orientation and inserted in the VIIL telomere. Levels of URA3 silencing were assessed as $\% F_{O A}{ }^{R}$ cells and plotted. Data is from Table 2. Statistical significance ( $p$ values) for the difference between the control construct (no ARS, middle of the graph) and the ARSs in URA3-B1-ACS-tel orientation are shown next to each bar. The $p$ values for the constructs in URA3-ACS-B1-tel orientation are significantly lower than 0.05 and are not shown.

telomere (or at any other telomere) the proportion of repressed $U R A 3$ within a cell population is modulated by the strength of the subtelomeric proto-silencer elements [20]. This proportion is easily assessed as \% FOA resistant cells ((FOA (5-fluoro-orotic acid) is converted to a toxin by the enzyme encoded by URA3)) [23]. Hence, in our assays the per cent FOA-resistant cells represents the proto-silencer strength of the engineered ARSs.

Parallel assays were conducted to test how the mutations in the $\underline{B 1}$ elements affect the replicator activity of the four $A \overline{R S S}$ and if these effects correlate to the decrease in $A R S$-driven silencing (Figure 3). To this end, all wild type and mutant $A R S$ fragments were subcloned in mini-chromosomes containing CEN4 and URA3 (Figure 3) and mini-chromosome stability assays were performed. $A R S / C E N$ mini-chromosomes are replicated once per cell cycle and are properly segregated during mitosis. Their normal loss rate is about 3-5\% per generation $[22,26]$. In our analyses any increase in the loss rate is indicative of malfunctioning of the origin. This assay provides a highly sensitive measure of the activity of the origins and has been instrumental in the deciphering of the regulatory elements in many $A R S$ 's.

\section{The orientation of $A R S$ s determines the level of telomeric silencing}

It has previously been shown that ARS317 exerts orientation-dependent silencing when the whole $H M R-E$ 
silencer is moved to the $H M L$ locus [16]. The possibility of directional silencing prompted us to establish an orientation in which all analysed ARSs would produce similar levels of URA3 silencing. ARS305, ARS605, ARS317 and ARS319 were sub-cloned in both directions ( $A D H 4$ URA3-ACS-B1-tel and ADH4-URA3-B1-ACS-tel) and then inserted between ADH4 and the VIIL telomere of W303 cells as described previously $[4,27,28]$. Consistent with their established role as proto-silencers [20], in the URA3ACS-B1-tel orientation all ARSs increased the proportion of FOA resistant $\left(\mathrm{FOA}^{\mathrm{R}}\right)$ cells to $67-81 \%$ relative to $36 \%$ in the construct with no ARS (Figure 1, upper part). No

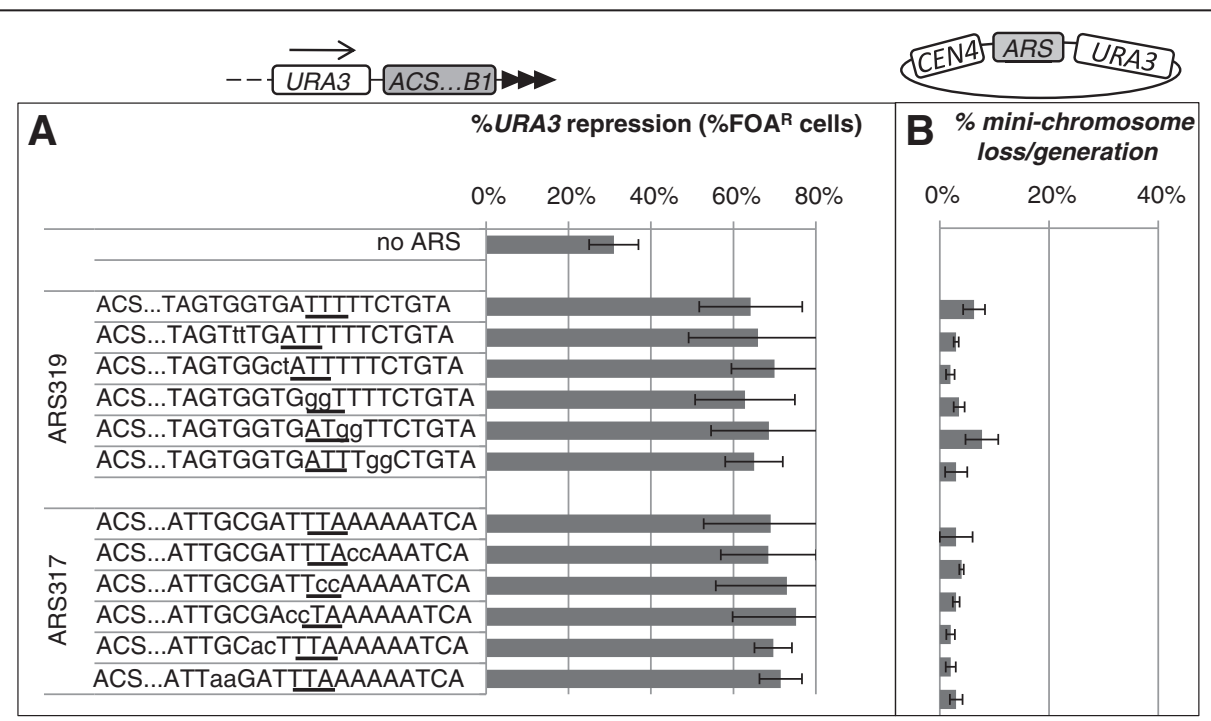

Figure 3 Proto-silencer and replicator activity of ARS317 and ARS319. A. The ARSS and their mutant derivatives (shown on the left) were integrated in the VIIL telomere in URA3-ACS-B1-tel direction. The putative WTW motifs are underlined. The mutations are depicted by small letters. The silencer activity of the constructs was assessed as \% FOA ${ }^{R}$ cells and plotted. Data is from Table 1. The $p$ values for all constructs are above 0.05 and are not shown. B. The ARSS and their mutant derivatives were cloned between CEN4 and URA3 to produce mini-chromosomes. All mini-chromosomes were transformed in W303 cells and loss per generation was calculated and plotted. Data is from Table 1. 
significant difference between silencer and replicator $A R S$ was observed. In the opposite URA3-B1-ACS-tel orientation ARS305 produced FOA ${ }^{\mathrm{R}}$ values comparable to the construct without any ARS (Figure 1, lower part). Remarkably, ARS317, ARS605 and especially ARS319 produced statistically significant decrease $(p<0.05)$ of silencing relative to the control thus clearly displaying anti-silencing properties. In summary, the orientation of $A R S$ relative to the telomere has a major impact on the level of subtelomeric silencing. From a technical point of view, in the URA3-ACS-B1-tel orientation all ARSs showed similar proportions of $\mathrm{FOA}^{\mathrm{R}}$ cells that allow for direct comparison of the role of their $\underline{B 1}$ elements in gene silencing.

\section{The same $\underline{B 1}$ elements in ARS305 and ARS605 contribute to gene silencing and DNA replication}

Next, we measured the contribution of the $B 1$ elements to DNA replication and to gene silencing. We mutagenized the replicators (ARS305 and ARS605) and silencers (ARS317 and ARS319) in the region encompassing their putative $\underline{B 1}$ (WTW) motifs by replacing two bases at a time (Figure 2A, Figure 3A). All wild type and mutant ARSs were cloned in ADH4-URA3-ACS-B1-tel orientation, inserted in the VIIL telomere and subjected to the FOA-resistance assays. In parallel, the same ARSS were cloned in URA3/CEN4 mini-chromosomes and tested for their replicator activity. The substitutions in the WTW motifs (shown by the rectangle in Figure 2A) of ARS305 and ARS605 caused statistically significant decrease in the proportion of $\mathrm{FOA}^{\mathrm{R}}$ cells relative to their non-mutated counterparts (Figure 2A). The flanking sequences in ARS305 had little effect, while in ARS605 they produced some minor reduction in silencer activity. In the mini-chromosome stability assay exactly the same mutations caused substantial increase of the loss per generation rates (Figure 2B). There is a good agreement in the magnitude of effects in the two assays with all mutants tested (Figure $2 \mathrm{~A}$ and $\mathrm{B}$ ). These observations indicate that the same $\underline{B 1}$ elements in ARS305 and ARS605 contribute to their silencer and replicator activity.

\section{Silencer $A R S s$ lack functionally identifiable $\underline{B 1}$}

Similar analyses of the two silencer ARSs (ARS317 and ARS319) showed that none of the two-base substitutions in the vicinity of the WTW element altered the levels of URA3 silencing (Figure 3A). To warrant for the existence of aberrantly positioned $\underline{B 1}$ we expanded the scanning substitutions as compared to ARS305 and ARS605, but no effects were observed. Similarly, the replicator activity of ARS317 and ARS319 remained largely unaffected by the mutations (Figure $3 \mathrm{~B}$ ). Only one of the ARS319 constructs showed modest increase in the loss rate of the mini-chromosome, but the mutation was outside of the canonical WTW element. Clearly, both assays failed to reveal a $\underline{B 1}$ element in ARS317 and ARS319.

\section{Discussion}

Lack of canonical $\underline{B 1}$ element

While the position and consensus of the $\underline{B 2}, \underline{B 3}$ and $\underline{B} 4$ auxiliary elements vary between different $\overline{A R S s}$, the $A C S-B 1$ module serves as a binary binding site for the association of ORC and seems highly conserved $[2,8,11,29,30]$. Indeed, recent studies have identified a strong WTW consensus 15-17 bases downstream of the core $A C S[8,13]$. Mutations in this $\underline{B 1}$ motif have caused significant loss of replicator activity in most ARSs [8,13], but its role in gene silencing has not been determined. Even more, mutations in the WTW consensus of one silencer ARS (ARS317) did not affect its replicator activity [8]. It seems conceivable that ARSs could utilize the WTW motif for replication and use an alternative $\underline{B 1}$ element for gene silencing [21]. It also seems possible that silencer and replicator ARSs have different $B 1$ elements and different type of interaction with ORC $[4,21]$. Here we have addressed both possibilities. We have shown that exactly the same mutations impair the replicator and the silencing function of the tested replicator ARSs (Figure 2). Hence, the answer to the first question is negative: these two functions are not determined by alternative $\underline{B 1}$ elements.

Surprisingly, the analyses of the two silencer ARSS (ARS317 and ARS319) have revealed that neither the replicator nor the silencer function was affected by any of the two-base substitutions within the 10 base region of the putative $\underline{B 1}$ (Figure 3). Again, we failed to obtain any evidence in favor of optional usage of $\underline{B 1}$ in silencing and replication. However, it is apparent that ARS317 and ARS319 have a different $\underline{B 1}$ or do not possess one at all. In the core $X$ and $Y^{\prime}$ subtelomeric elements there are more than 100 close matches to ARS319 with high levels of homology in their ACS-B1 module (Figure 4B). We suggest that all these plus $A R S 317$ represent a subclass of $A R S$ with a novel type of $\underline{B 1}$ element or with no $\underline{B 1}$ element.

ARS display considerable diversity of the sequences surrounding the core $A C S[8,13,31]$. To this diversity we add the extreme case of lack of a canonical $B 1$. This is an intriguing issue. Being a secondary site for ORC binding, $B 1$ is expected to be important if not essential. It is possible that we have identified no $\underline{B 1}$ element because in silencer ARS there is no second site of association for ORC. For example, a specialized extended 17 base pair EACS $[7,8,12]$ could provide a single highaffinity site for the binding of ORC and minimize the significance of the $\underline{B 1}$ element. Alternatively, these ACS$B 1$ sites are bi-partite, but $\underline{B 1}$ is broader. If this is the 


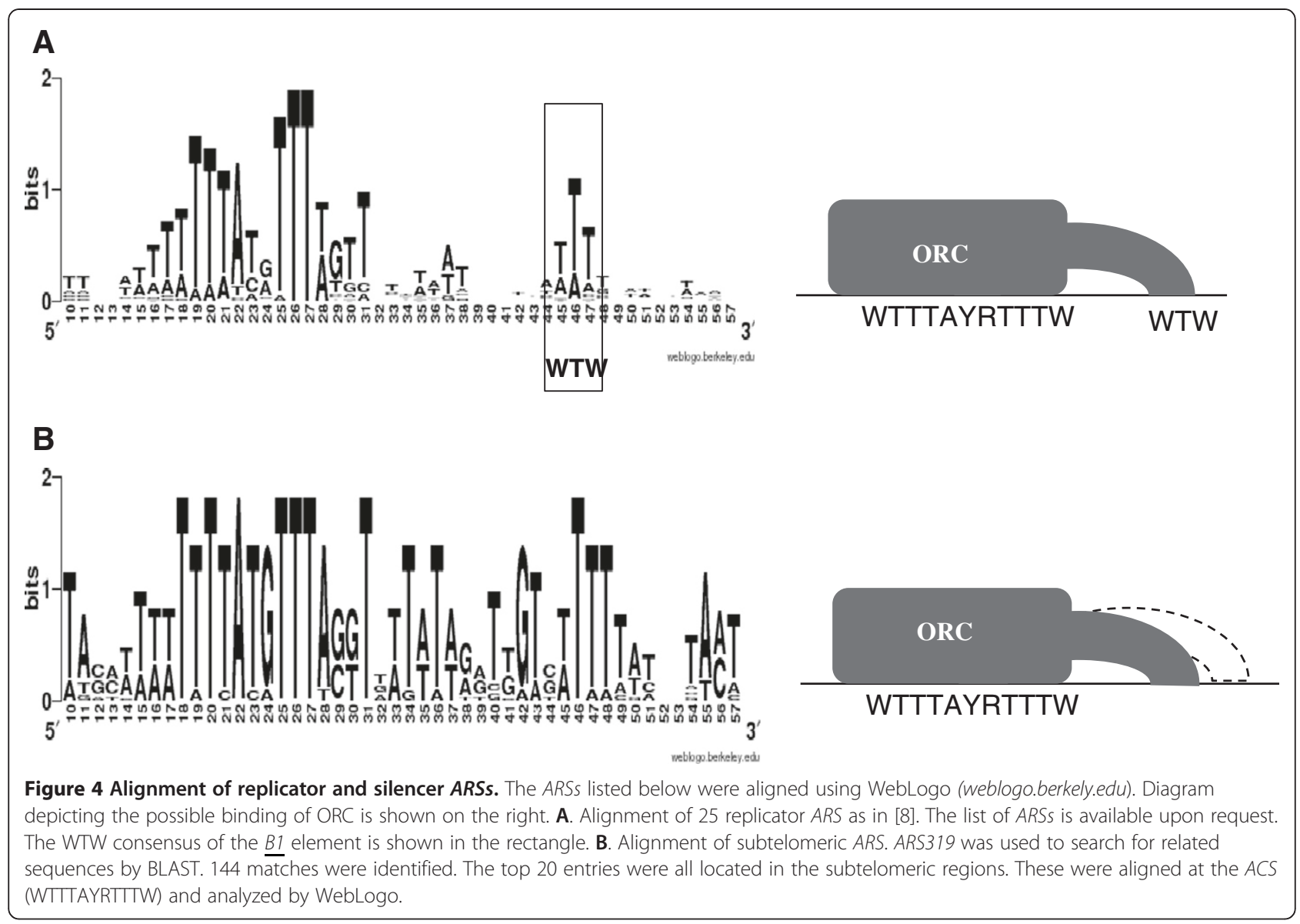

case, the two-base substitutions that normally destroy the WTW in replicator ARSS will not work on ARS319 and ARS317. Indeed, the alignment of ARS319 and other telomeric ARSs shows more than one potential WTW site in the area of $B 1$ (Figure 4B). The same applies to ARS317 (not shown). However, the two ARS from the $H M L$ mating type locus do not show significant similarity to the putative $\underline{B 1}$ regions of ARS319 and ARS317 (not shown). Even more, the high overall homology of sub-telomeric ARSs (Figure 4B) precludes the recognition of a different $\underline{B 1}$ even if it existed. Hence, while a broader $\underline{B 1}$ in silencer $A R S$ remains possible, a consensus is difficult to identify. Ultimately, it is possible that a combination of an extended ACS [12,13,31] and broader $\underline{B 1}$ provide alternatives for the binding of ORC that are hard to unveil by alignment algorithms.

Within eukaryotes, budding yeasts are the only known species with well defined sequences of origins and well defined binding sites for ORC. In all other species the origins are quite dissimilar and are recognizable only as $\mathrm{A} / \mathrm{T}$ rich regions of DNA. This study together with other recent studies $[8,12,13]$ suggests a certain level of diversity between different ARS in budding yeast. The significance of this diversity is yet to be determined.

\section{Directionality of silencing}

Silencers and proto-silencer in S. cerevisiae are ciselements that serve as focal points for the recruitment of Sir proteins [1] and in most cases are thought to act in bi-directional fashion. However, while selecting the optimal conditions for our silencing assays we came across strong and reproducible orientation-dependent effects. All ARSs were potent proto-silencers only in the URA3-ACS-B1-tel orientation, the same orientation found in natural sub-telomeric ARS elements. In the opposite URA3-B1-ACS-tel orientation ARS319 displayed strong anti-silencing activity while ARS317 and ARS605 caused modest de-repression of URA3. These experiments have not been developed to establish if the ACS$B 1$ module or other unknown sites within the cloned fragments exert these directional effects. However, several arguments support the idea that $A C S-B 1$ could play a central role. Earlier research has acquired evidence for directional silencing by $H M R-E$ when it is inserted in the $H M L$ locus [15,17]. HMR-E consists of ARS317 and binding sites for Abf1p and Rap1p. Gene repression has been robust at the Abf1p binding site of $H M R-E$ and weak at the $A C S$ side [15]. A stably positioned nucleosome was found adjacent to the ACS site of ARS317 and 
not on the side containing the $B 1$ element [15]. The binding sites for Abf1p and Rap1p also contribute to the directionality of $H M R-E$. It is noteworthy that, excluding $A R S 317$, none of our other constructs contains identifiable Abf1p or Rap1p binding sites thus leaving ACS-B1 as a likely candidate for the effects we have observed. In addition, ARS319 and not ARS317 displayed the strongest directional effects (Figure 1). If $A C S-B 1$ is the key directional element, a stably positioned nucleosome next to ACS can stimulate the transfer of Sir proteins approaching from the telomere thus acting as a relay point. In the opposite orientation, a nucleosome-free DNA generated by the association of ORC [32] could prevent Sir protein spreading and act as a chromatin insulator [33]. However, why nucleosome-free DNA on one side of $A R S$ would work as insulator in one orientation is yet to be established. Structural studies on ORC bound to different $A C S-B 1$ modules can address this possibility.

\section{Conclusions}

All tested ARS display proto-silencing activity in the $A C S-B 1$-tel orientation relative to the telomere. However, in the B1-ACS-tel orientation ARS305 does not show proto-silencing activity, while ARS605, ARS317, ARS319 display anti-silencing activity. Hence, there is a strong orientation dependency in the proto-silencing activity of ARS. In addition, ARS317 and ARS319 do not possess a canonical $\underline{B 1}$ element thus suggesting a different ACS-B1 module relative to ARS305, ARS605 and numerous replicator $A R S$.

\section{Materials and methods}

\section{Constructs}

ARS305, ARS605, ARS317 and ARS319 were amplified by PCR from the genomic DNA of W303 strain using Phusion polymerase (NEB) according to the instructions of the manufacturer. The genomic coordinates (as per the updates available in January 2010) of the amplified fragments are as follows: ARS305, III: 39392-39774; ARS605, VI: 135860-136202; ARS317, III: 292894292369; ARS319, III: 315639-315989. The primer sequences are available upon request. The amplified fragments were sub-cloned in the BamH1 site of pUCAIV [27] between the telomeric $\mathrm{TG}_{1-3}$ repeats and the URA3 reporter. Our constructs do not contain any additional sub-telomeric elements. Two bases at a time were replaced in the vicinity of the $\underline{B 1}$ elements of the

Table 1 Silencer and replicator activity of mutated ARSs

\begin{tabular}{|c|c|c|c|c|c|c|c|}
\hline & & \multicolumn{3}{|c|}{ Silencer activity (FOA-resistance assay) } & \multicolumn{3}{|c|}{ Replicator activity (mini-chromosome stability assay) } \\
\hline & & FOA $^{R}$ & & STD & Loss/generation & & STD \\
\hline & no ARS & $31 \%$ & $(n=9)$ & $6 \%$ & $\mathrm{n} / \mathrm{a}$ & & \\
\hline \multirow[t]{5}{*}{ ARS305 } & ACS. ..ATGTTTATTTTCCCTTTAA & $66 \%$ & $(n=9)$ & $2 \%$ & $3 \%$ & $(n=9)$ & $0 \%$ \\
\hline & ACS. . .АTGTTTATTTCACCTTTAA & $69 \%$ & $(n=9)$ & $8 \%$ & $4 \%$ & $(n=9)$ & $1 \%$ \\
\hline & ACS. . .АTGTTTATСCTCCCTTTAA & $46 \%$ & $(n=9)$ & $7 \%$ & $30 \%$ & $(n=9)$ & $6 \%$ \\
\hline & ACS...ATGTTTCCTTTCCCTTTAA & $38 \%$ & $(n=9)$ & $16 \%$ & $30 \%$ & $(n=9)$ & $9 \%$ \\
\hline & ACS. . .ATGTCCATTTTCCCTTTAA & $69 \%$ & $(n=9)$ & $7 \%$ & $2 \%$ & $(n=9)$ & $0 \%$ \\
\hline \multirow[t]{5}{*}{ ARS605 } & ACS. . AGGAAACTTTACAAAGCGT & $64 \%$ & $(n=6)$ & $11 \%$ & $4 \%$ & $(n=6)$ & $1 \%$ \\
\hline & ACS. . AGGAAACTTTAacAAGCGT & $53 \%$ & $(n=6)$ & $4 \%$ & $19 \%$ & $(n=6)$ & $2 \%$ \\
\hline & ACS. . AGGAAACTTCCCAAAGCGT & $35 \%$ & $(n=6)$ & $12 \%$ & $40 \%$ & $(n=6)$ & $5 \%$ \\
\hline & ACS. . AGGAAACCCTACAAAGCGT & $56 \%$ & $(n=6)$ & $8 \%$ & $19 \%$ & $(n=6)$ & $2 \%$ \\
\hline & ACS. . AGGAACaTTTACAAAGCGT & $64 \%$ & $(n=6)$ & $20 \%$ & $9 \%$ & $(n=6)$ & $6 \%$ \\
\hline \multirow[t]{6}{*}{ ARS319 } & ACS...TAGTGGTGATTTTTCTGTA & $64 \%$ & $(n=12)$ & $13 \%$ & $6 \%$ & $(n=9)$ & $2 \%$ \\
\hline & ACS...TAGTttTGATTTTTCTGTA & $66 \%$ & $(n=12)$ & $17 \%$ & $3 \%$ & $(n=9)$ & $0 \%$ \\
\hline & ACS. .TAGTGGCtATTTTTCTGTA & $70 \%$ & $(n=12)$ & $11 \%$ & $2 \%$ & $(n=9)$ & $1 \%$ \\
\hline & ACS. . .TAGTGGTGggTTTTCTGTA & $63 \%$ & $(n=12)$ & $12 \%$ & $4 \%$ & $(n=9)$ & $1 \%$ \\
\hline & ACS. . .TAGTGGTGATggTTCTGTA & $69 \%$ & $(n=12)$ & $14 \%$ & $8 \%$ & $(n=9)$ & $3 \%$ \\
\hline & ACS. .TAGTGGTGATTTggCTGTA & $65 \%$ & $(n=12)$ & $7 \%$ & $3 \%$ & $(n=9)$ & $2 \%$ \\
\hline \multirow[t]{6}{*}{ ARS317 } & ACS. . .ATTGCGATTTAAAAAATCA & $69 \%$ & $(n=9)$ & $16 \%$ & $3 \%$ & $(n=9)$ & $3 \%$ \\
\hline & ACS. . .ATTGCGATTTACCAAATCA & $68 \%$ & $(n=9)$ & $12 \%$ & $4 \%$ & $(n=9)$ & $0 \%$ \\
\hline & ACS. . ATTGCGATTCCAAAAATCA & $73 \%$ & $(n=9)$ & $17 \%$ & $3 \%$ & $(n=9)$ & $1 \%$ \\
\hline & ACS. . ATTGCGACCTAAAAAATCA & $75 \%$ & $(n=9)$ & $15 \%$ & $2 \%$ & $(n=9)$ & $1 \%$ \\
\hline & ACS. . ATTGCaCTTTAAAAAATCA & $70 \%$ & $(n=9)$ & $5 \%$ & $2 \%$ & $(n=9)$ & $1 \%$ \\
\hline & ACS...ATTaaGATTTAAAAAATCA & $71 \%$ & $(n=9)$ & $5 \%$ & $3 \%$ & $(n=9)$ & $1 \%$ \\
\hline
\end{tabular}


Table 2 Silencer activity of ARSs cloned in opposite orientations

\begin{tabular}{|c|c|c|c|}
\hline \multicolumn{4}{|c|}{ Silencer activity (FOA-resistance assay) } \\
\hline & $\mathrm{FOA}^{\mathrm{R}}$ & & $\overline{\text { STD }}$ \\
\hline no ARS & $36 \%$ & $(n=9)$ & $4 \%$ \\
\hline ...URA3-ACS319-B1-tel & $81 \%$ & $(n=9)$ & $6 \%$ \\
\hline ...URA3-ACS317-B1-tel & $77 \%$ & $(n=9)$ & $5 \%$ \\
\hline ...URA3-ACS305-B1-tel & $78 \%$ & $(n=9)$ & $12 \%$ \\
\hline ...URA3-ACS605-B1-tel & $67 \%$ & $(n=9)$ & $11 \%$ \\
\hline ...URA3-B1-ACS319-tel & $7 \%$ & $(n=9)$ & $7 \%$ \\
\hline ...URA3-B1-ACS317-tel & $21 \%$ & $(n=9)$ & $11 \%$ \\
\hline ...URA3-B1-ACS305-tel & $47 \%$ & $(n=9)$ & $7 \%$ \\
\hline ...URA3-B1-ACS605-tel & $26 \%$ & $(n=9)$ & $8 \%$ \\
\hline
\end{tabular}

ARSs by site-directed mutagenesis. The mutated sequences are shown in Table 1. All mutations have been confirmed by DNA sequencing. The nucleotide sequences of the primers used to amplify genomic DNA and to mutate the cloned fragments are available upon request.

\section{Growth media and conditions}

W303 cells (ade2-1 trp1-1 can1-100 leu2-3,112 his311,15 ura3-1) were routinely grown on rich medium (YPD) at $23^{\circ} \mathrm{C}$. Cells transformed with URA3 integrating fragments or URA3/CEN4/ARS mini-chromosomes were selected on Synthetic Complete (SC) medium without uracil. Cells with repressed URA3 were selected on SC medium supplemented with $1 \mathrm{~g} / \mathrm{l}$ Fluoro-Orotic Acid (FOA) (Toronto Chemicals).

\section{Telomere position effect (TPE) assays}

Fragments containing ADH4, URA3, ARS and telomeric $T G_{1-3}$ repeats were released by digestion of pUCAIV derivatives with SalI and EcoRI and used to transform W303 cells. This treatment efficiently integrates the constructs between ADH4 and the VIIL telomere [27]. Telomeric integration was confirmed by PCR and variegated expression of URA3 [27]. To warrant the loss of unintegrated constructs (these are linear DNAs lacking CEN elements), transformants were re-streaked on Scura and SC/FOA plates and then an isolated colony from the SC-ura plate was grown for 20 generations in nonselective (YPD) medium. Serial 1:10 dilutions were prepared and $5 \mu \mathrm{l}$ aliquots were spotted on $\mathrm{SC}$ and $\mathrm{SC} / \mathrm{FOA}$ plates. The $\% \mathrm{FOA}^{\mathrm{R}}$ for each independent culture was calculated. Each construct was analysed in triplicate (three independent colonies per transformation) in three or more independent transformations. The average values and standard deviation from these experiments were calculated in Microsoft Excel and are shown in Tables 2 and 1.

\section{Mini-chromosome stability assay}

The cloned and mutated ARSs were released from pUCAIV by digestion with BamH1 and sub-cloned in a pUC119 based mini-chromosome [2] containing URA3 and CEN4. Each mini-chromosome was independently transformed in W303 cells. Three colonies were isolated from SC-ura plates, re-streaked on SC-ura plates and suspended in non-selective (YPD) medium. The cultures were grown for 20 generations in non-selective YPD medium. Serial 1:10 dilutions of the cultures prior and after growth in non-selective medium were prepared and $5 \mu \mathrm{l}$ aliquots were spotted on SC and SC-ura plates. The per cent of ura+ cells prior and after growth in nonselective medium were used to calculate the minichromosome loss per generations as in [22,26]. Each mini-chromosome was analysed in triplicate (three independent colonies per transformation) in three independent transformations. The average values and standard deviation were calculated in Microsoft Excel and are shown in Table 2.

\section{Abbreviations}

W: A/T; Y: C/T; R: A/C; ARS: Autonomously Replicating Sequences; ACS: ARS Consensus Sequence; ORC: Origin Recognition Complex; FOA: 5-fluoro-orotic acid; TPE: Telomere Position Effect.

\section{Competing interests}

The authors declare that they have no competing interests.

\section{Authors' contributions}

PC-R conceived and participated in the design of the study, cloned and mutagenized the analyzed ARSs, carried out the silencing and some of the replicator experiments and drafted the manuscript. SP and KS carried out the replicator assays and prepared figures. KY conceived and participated in the design of the study and wrote the manuscript. All authors have read and approved the manuscript.

\section{Acknowledgements}

This study was supported by a grant from NSERC (\#217548-2010) to KY. We thank Dr. Gottschling and Dr. Li for gift of the pUCAIV and the pUC119/ CEN4/URA3 plasmids. PC-R, SP and KS thank Daniel Jeffery for expert support and advise during the study.

\section{Author details}

${ }^{1}$ Department of Molecular and Cellular Biology, University of Guelph, Guelph, ON, Canada. ${ }^{2}$ Current address: Department of Medical Genetics, Molecular Epigenetics Group, University of British Columbia, Vancouver, Canada.

Received: 11 September 2012 Accepted: 15 November 2012 Published: 16 November 2012

\section{References}

1. Rusche $L N$, Kirchmaier $A L$, Rine J: The establishment, inheritance, and function of silenced chromatin in Saccharomyces cerevisiae. Annu Rev Biochem 2003, 72:481-516.

2. Marahrens $Y$, Stillman B: A yeast chromosomal origin of DNA replication defined by multiple functional elements. Science 1992, 255(5046):817-823.

3. Rao H, Marahrens $Y$, Stillman B: Functional conservation of multiple elements in yeast chromosomal replicators. Mol Cell Biol 1994, 14(11):7643-7651.

4. Rehman MA, Wang D, Fourel G, Gilson E, Yankulov K: Subtelomeric ACS-containing proto-silencers act as antisilencers in replication factors mutants in Saccharomyces cerevisiae. Mol Biol Cell 2009, 20(2):631-641.

5. Lee DG, Bell SP: Architecture of the yeast origin recognition complex bound to origins of DNA replication. Mol Cell Biol 1997, 17(12):7159-7168. 
6. Rao H, Stillman B: The origin recognition complex interacts with a bipartite DNA binding site within yeast replicators. Proc Natl Acad Sci USA 1995, 92(6):2224-2228.

7. Theis JF, Newlon CS: The ARS309 chromosomal replicator of Saccharomyces cerevisiae depends on an exceptional ARS consensus sequence. Proc Natl Acad Sci USA 1997, 94(20):10786-10791.

8. Chang F, Theis JF, Miller J, Nieduszynski CA, Newlon CS, Weinreich M: Analysis of chromosome III replicators reveals an unusual structure for the ARS318 silencer origin and a conserved WTW sequence within the origin recognition complex binding site. Mol Cell Biol 2008, 28(16):5071-5081.

9. Wilmes GM, Bell SP: The B2 element of the Saccharomyces cerevisiae $A R S 1$ origin of replication requires specific sequences to facilitate pre-RC formation. Proc Natl Acad Sci USA 2002, 99(1):101-106.

10. Zou L, Stillman B: Assembly of a complex containing Cdc45p, replication protein $A$, and $M c m 2 p$ at replication origins controlled by S-phase cyclin-dependent kinases and Cdc7p-Dbf4p kinase. Mol Cell Biol 2000, 20(9):3086-3096.

11. Theis JF, Newlon CS: Domain B of ARS307 contains two functional elements and contributes to chromosomal replication origin function. Mol Cell Biol 1994, 14(11):7652-7659.

12. Palacios DeBeer MA, Muller U, Fox CA: Differential DNA affinity specifies roles for the origin recognition complex in budding yeast heterochromatin. Genes Dev 2003, 17(15):1817-1822.

13. Chang F, May CD, Hoggard T, Miller J, Fox CA, Weinreich M: Highresolution analysis of four efficient yeast replication origins reveals new insights into the ORC and putative MCM binding elements. Nucleic Acids Res 2011, 39(15):6523-6535.

14. Weinreich M, Liang C, Chen HH, Stillman B: Binding of cyclin-dependent kinases to ORC and Cdc6p regulates the chromosome replication cycle. Proc Natl Acad Sci USA 2001, 98(20):11211-11217.

15. Zou Y, Yu Q, Bi X: Asymmetric positioning of nucleosomes and directional establishment of transcriptionally silent chromatin by Saccharomyces cerevisiae silencers. Mol Cell Biol 2006, 26(20):7806-7819.

16. Zou Y, Yu Q, Chiu YH, Bi X: Position effect on the directionality of silencer function in Saccharomyces cerevisiae. Genetics 2006, 174(1):203-213.

17. Bi X, Braunstein M, Shei GJ, Broach JR: The yeast HML I silencer defines a heterochromatin domain boundary by directional establishment of silencing. Proc Natl Acad Sci USA 1999, 96(21):11934-11939.

18. Walmsley RW, Chan CS, Tye BK, Petes TD: Unusual DNA sequences associated with the ends of yeast chromosomes. Nature 1984 310(5973):157-160.

19. Chan CS, Tye BK: Organization of DNA sequences and replication origins at yeast telomeres. Cell 1983, 33(2):563-573.

20. Fourel G, Lebrun E, Gilson E: Protosilencers as building blocks for heterochromatin. Bioessays 2002, 24(9):828-835.

21. Rehman MA, Yankulov K: The dual role of autonomously replicating sequences as origins of replication and as silencers. Curr Genet 2009, 55(4):357-363.

22. Tye BK: Minichromosome maintenance as a genetic assay for defects in DNA replication. Methods 1999, 18(3):329-334.

23. Yankulov K: Dare to challenge the silence? Telomeric gene silencing revisited. Nucleus 2011, 2(6):513-516.

24. Ottaviani A, Gilson E, Magdinier F: Telomeric position effect: from the yeast paradigm to human pathologies? Biochimie 2008, 90(1):93-107.

25. Rusche LN, Kirchmaier AL, Rine J: Ordered nucleation and spreading of silenced chromatin in Saccharomyces cerevisiae. Mol Biol Cell 2002, 13(7):2207-2222

26. Kramer DJ, Gauthier L, Yankulov K: Higher-accuracy method for measuring minichromosome stability in Saccharomyces cerevisiae. Biotechniques 2002, 32(5):1036-1040.

27. Gottschling DE, Aparicio OM, Billington BL, Zakian VA: Position effect at S. cerevisiae telomeres: reversible repression of Pol II transcription. Cell 1990, 63(4):751-762.

28. Fourel G, Revardel E, Koering CE, Gilson E: Cohabitation of insulators and silencing elements in yeast subtelomeric regions. EMBO J 1999, 18(9):2522-2537.

29. Huang RY, Kowalski D: Multiple DNA elements in ARS305 determine replication origin activity in a yeast chromosome. Nucleic Acids Res 1996, 24(5):816-823

30. Stillman B: Origin recognition and the chromosome cycle. FEBS Lett 2005, 579(4):877-884.
31. Weinreich M, Palacios DeBeer MA, Fox CA: The activities of eukaryotic replication origins in chromatin. Biochim Biophys Acta 2004, 1677(1-3):142-157.

32. Lipford JR, Bell SP: Nucleosomes positioned by ORC facilitate the initiation of DNA replication. Mol Cell 2001, 7(1):21-30.

33. Murrell A: Setting up and maintaining differential insulators and boundaries for genomic imprinting. Biochem Cell Biol 2011, 89(5):469-478.

doi:10.1186/1471-2199-13-34

Cite this article as: Chisamore-Robert et al: Directional telomeric silencing and lack of canonical B1 elements in two silencer Autonomously Replicating Sequences in S. cerevisiae. BMC Molecular Biology 2012 13:34.

\section{Submit your next manuscript to BioMed Central and take full advantage of:}

- Convenient online submission

- Thorough peer review

- No space constraints or color figure charges

- Immediate publication on acceptance

- Inclusion in PubMed, CAS, Scopus and Google Scholar

- Research which is freely available for redistribution

Submit your manuscript at www.biomedcentral.com/submit
C Biomed Central 